\title{
Nutraceuticals and separations
}

\author{
Luigi Mondello
}

Published online: 9 March 2013

(C) Springer-Verlag Berlin Heidelberg 2013

The term "nutraceutical" was originally defined by Stephen L. DeFelice, founder and chairman of the Foundation for Innovation in Medicine (Crawford, NJ, USA, 1989), as a combination of the words "nutrition" and "pharmaceutical": it refers to nutritional products which have effects that are relevant to health. In contrast to pharmaceuticals, however, they are not synthetic substances or chemical compounds formulated for specific indications. According to the European Nutraceutical Association, nutraceuticals are "naturally derived bioactive compounds that are found in foods, dietary supplements and herbal products, and have health promoting, disease preventing, or medicinal properties."

For centuries, people have turned to natural remedies for the prevention and treatment of a variety of common ailments and diseases; with the advent of modern medicine, natural remedies have taken a back seat, giving way to a blooming chemical industry. More recently, the search for less expensive treatments, and the undesirable aggressive side effects often associated with synthetic molecules, have resulted in a turnabout, making the popularity of nutraceuticals soar. As a consequence, the global nutraceutical market was worth about $\$ 151$ billion in 2011 , and it is estimated it will be worth about $\$ 207$ billion by 2016 , with a projected compound annual growth rate of $6.5 \%$.

Such products may range from isolated nutrients, dietary supplements, and specific diets to genetically engineered foods, herbal products, and processed foods such as cereals,

Published in the topical collection Nutraceuticals and Separations with guest editor Luigi Mondello.

L. Mondello

University of Messina, Viale Annunziata,

98168 Messina, Italy

\section{Mondello ( $ه)$}

Università Campus Bio-Medico di Roma,

Via Àlvaro del Portillo 21,

00128 Rome, Italy

e-mail: 1mondello@unime.it soups, and beverages. Nutraceutical categories include dietary supplements (e.g., vitamins, minerals, coenzyme Q, carnitine, and botanicals such as ginseng and gingko biloba), functional foods (e.g., oats, bran, prebiotics, omega-3, plant sterols, and stanols), and medicinal foods (e.g., transgenic plants, health bars with added medications). The best known physiological benefits or protection against chronic disease include antioxidant activity (resveratrol, flavonoids), reduction of hypercholesterolemia (soluble dietary fibers), cancer prevention (broccoli, fiddleheads), and protection against cardiovascular disease ( $\alpha$-linolenic acid).

"Natural" is not synonymous with "safe" however, and a cost-effective and scientifically based framework is needed to evaluate both the safety and the effectiveness of these items. Medical researchers need to merge and integrate information from clinical studies into responsible medical practice, and, on the other hand, industry, professional organizations, academia, and health regulatory agencies need to add specific legal and scientific criteria to the definition and standards for nutraceuticals. For their part, analysts are challenged with the development of suitable separation techniques which are capable of providing the resolution and specificity of detection required. Proper knowledge of the qualitative composition and contents of these natural sources is mandatory information for rational consumption and correlation of beneficial effects to dietary intake. An additional issue related to nutraceuticals is that the composition and contents of active constituents in natural sources may vary depending on several factors (climate, season, soil, etc.), and as a consequence it is critical to assess uniform quality, quantification, and standardization.

The use of advanced analytical techniques is, therefore, mandatory in all the steps involved in nutraceutical research, from the identification and characterization of new molecules, quantification of their amount in natural sources, and assay of their biological activity to product formulation, quality control, and clinical studies. Given that the complexity of these matrices requires advanced separation/ 
identification power, the choice of the analytical technique will ultimately depend on the type of target compounds, and the natural source in which they are present.

Gas chromatography (GC) and high-performance liquid chromatography (LC) are most widespread separation tools for this purpose, because of their versatility and easy operation, but capillary electrophoresis (CE) has also proven to be effective. These techniques are typically used in a combined way with mass spectrometry (MS), nuclear magnetic resonance (NMR) spectroscopy, Fourier-transform infrared (FTIR) spectroscopy, and other detection methods. Size, polarity, thermal stability, and other properties of the sample will affect the choice of the separation technique (GC, highperformance LC, CE), and the type of detector employed (MS, NMR, FTIR, flame ionization, fluorescence).

Another aspect of considerable importance when dealing with nutraceuticals is the need for appropriate, fast, and cost-effective extraction processes able to isolate the compounds of interest. In this regard, advanced extraction techniques such as pressurized liquid extraction and QuEChERS (quick, easy, cheap, effective, rugged, and safe) have demonstrated superior performance over conventional liquidliquid extraction methods.

One more facet in ensuring a safe product is analysis of the final product before distribution; especially the measurement of toxic metals or metals intended to be present for quality and labeling confirmation may be required. Metals may be included in organic structures, or may derive from contamination through natural products, or in the manufacturing process. Their determination requires sensitive and specific detection to afford reliable quantitative data at low concentrations in a variety of matrices. In this regard, inductively coupled plasma MS, triple-quadrupole MS, and pulsed electrochemical detection have proven to be effective tools in measuring a variety of hazardous elements. Although the separation afforded can be enhanced by the use of high-performance stationary phases (e.g., partially porous particles) or multidimensional and comprehensive chromatography techniques (e.g., LC-GC, $\mathrm{LC} \times \mathrm{LC}, \mathrm{GC} \times \mathrm{GC}$ ), the scarcity of commercial pure standards still often hinders rapid and reproducible analysis of these compounds.
The emerging nutraceuticals industry is likely to occupy the landscape of the new millennium, and in this scenario nutritionists, toxicologists, and regulatory agencies are supposed to work strategically within an integrated network to scientifically validate their health effects and plan strict controls. Analysts, for their part, will be required to develop advanced analytical approaches to make identification and determination of nutraceutical compounds easier and more reliable, and to provide data to be merged and interconnected with data from well-designed clinical trials.

In this issue, some recent advances in the field of nutraceutical compounds are presented, including biological, analytical, and safety issues of concern in nutraceutical preparation and analysis. Topics of interest include the measurement of carbohydrates, carotenoids, procyanidins, furanocoumarins, anthocyanins, polyphenols, lipids, and sterols.

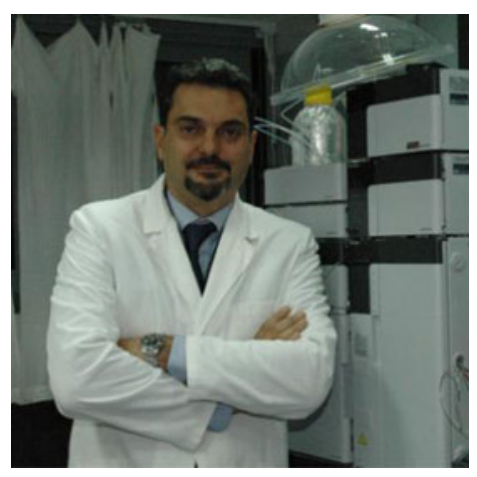

Luigi Mondello is Full Professor of Analytical Chemistry at the University of Messina, Italy, and Campus Bio-Medico University in Rome, Italy. He is the author of 200 scientific articles, 29 book chapters, and 11 reviews, he is the editor and co-editor of two books. He was chairman of the $36^{\text {th }}$ International Symposium on Capillary Chromatography, the $9^{\text {th }} \mathrm{GC} \times \mathrm{GC}$ Symposium (Riva del Garda, Italy, 2012), and the $14^{\text {th }}$ International Symposium on Advances in Extraction Technologies (Messina, Italy, 2012). He is a member of the Steering Committee of the Italian Separation Science Group of the Italian Chemical Society, a member of the Chromedia (Chromatography Knowledge Base) expert team, and co-president and co-founder of the Mediterranean Separation Science Foundation Training and Research Center. His research interests include the development of multidimensional and comprehensive techniques (LC-GC-MS, GC$\mathrm{GC}, \mathrm{GC} \times \mathrm{GC}, \mathrm{LC} \times \mathrm{LC}, \mathrm{LC} \times \mathrm{GC})$, and their application for the analysis of natural complex matrices. 